02;09;15

\title{
Анализ пространственного разрешения эксперимента по проверке принципа эквивалентности для нейтрона дифракционным методом
}

\author{
(C) В.В. Воронин ${ }^{1,2,3}$, Я.А. Бердников ${ }^{2}$, А.Я. Бердников ${ }^{2}$, \\ Ю.П. Брагинец ${ }^{1,2}$, И.А. Кузнецов ${ }^{1}$, М.В. Ласица ${ }^{1,2}$, \\ С.Ю. Семенихин ${ }^{1, \uparrow, ~ В . В . ~ Ф е д о р о в ~}{ }^{1,2,3}$
}

${ }^{1}$ НИЦ „Курчатовский институт“ ПИЯФ, Гатчина

${ }^{2}$ Санкт-Петербургский политехнический университет им. Петра Великого

${ }^{3}$ Санкт-Петербургский государственный университет

ฯ E-mail: semenikhin_sy@pnpi.nrcki.ru

Поступило в Редакцию 8 ноября 2016 г.

Проведены исследования пространственного разрешения установки по проверке эквивалентности инертной и гравитационной масс нейтрона методом дифракции в совершенном кристалле. Исследования проводились при углах Брэгга $(74-82)^{\circ}$. Обнаружено снижение пространственного разрешения при углах Брэгга $>78^{\circ}$.

DOI: $10.21883 /$ PJTF.2017.05.44365.16562

В настоящее время готовится эксперимент по проверке эквивалентности инертной и гравитационной масс нейтрона новым методом, основанным на использовании эффектов, возникающих при дифракции нейтронов в больших совершенных кристаллах [1]. Несмотря на то что для макроскопических тел принцип эквивалентности проверен с очень высокой точностью (см., например, [2]), на вопрос о его применимости к квантово-механическим объектам однозначного ответа нет $[3,4]$. В частности, к нарушению принципа эквивалентности могут приводит так называемые хамелеонные скалярные поля, предлагаемые для объяснения темной энергии во Вселенной [5]. Величина этого нарушения может зависеть от локальной плотности материи и, как следствие, от размера исследуемого объекта, поэтому справедливость принципа эквивалентности для макроскопических объектов не означает отсутствия возможности его нарушения для элементарных частиц [6]. 
В настоящее время выполнен ряд экспериментов по проверке принципа эквивалентности для нейтрона. Наиболее точное ограничение на отличие гравитационной массы нейтрона от инертной получено на уровне $2 \cdot 10^{-4}$ в работе [7], однако имеются сомнения в корректности некоторых выводов этой работы. Эксперимент с ультрахолодными нейтронами [8] позволил получить точность на уровне $10^{-3}$, и есть перспективы по ее существенному улучшению. Отличие предлагаемого метода от упомянутых выше заключается в прямой компенсации гравитационной силы, действующей на нейтрон, силой инерции, действующей на нейтрон в неинерциальной системе координат, связанной с Землей. По сути, эксперимент аналогичен классическим экспериментам Этвеша [9] или Брагинского [10], только в нашем случае на одной чаше весов находится Земля, а на другой - нейтрон.

Распространение нейтрона в кристалле в направлениях, близких к брэгговским, можно описать двумя типами блоховских волн $\psi^{(1)}$ и $\psi^{(2)}$, которые распространяются вдоль кристаллографических плоскостей, причем нейтроны в состоянии $\psi^{(1)}$ сконцентрированы преимущественно на ядерных плоскостях (максимумах ядерного потенциала), а в состоянии $\psi^{(2)}$ - между ними. По этой причине нейтроны в этих состояниях находятся в разных потенциалах и имеют слегка разные кинетические энергии. В симметричной схеме дифракции по Лауэ (входная грань кристалла перпендикулярна отражающим плоскостям) при точном выполнении условия Брэгга оба состояния возбуждаются с одинаковой вероятностью, и плотности тока нейтронов в обоих состояних направлены точно вдоль отражающих плоскостей. Отклонение от условия Брэгга приводит к отклонению направлений токов в противоположные стороны (рис. 1).

Траектории (линии, касательные к которым направлены по плотности тока) нейтрона в деформированном кристалле можно описать действием „сил Като“ [11], которые определяются степенью деформации кристалла и связанным с ней отклонением от условия Брэгга. Уравнение траектории имеет вид [11] (рис. 1):

$$
\frac{\partial^{2} z}{\partial y^{2}}= \pm \frac{\tan \left(\theta_{\mathrm{B}}\right)}{m_{0}} f_{k}(y, z),
$$

где $f_{k}(y, z)$ - сила Като, определяемая деформацией кристалла:

$$
f_{k}(y, z)=-\frac{k_{0}}{4 \cos \theta_{\mathrm{B}}}\left(\frac{\partial}{\partial z}+\frac{1}{c} \frac{\partial}{\partial y}\right) \alpha(y, z),
$$

Письма в ЖТФ, 2017, том 43, вып. 5 
$k_{0}-$ волновой вектор нейтрона в кристалле, $\theta_{\mathrm{B}}-$ угол Брэгга,

$$
\alpha(y, z)=\frac{g(y, z)^{2}+2\left(\mathbf{k}_{0} \cdot \mathbf{g}(y, z)\right)}{k_{0}^{2}}
$$

- параметр отклонения от точного условия Брэгга.

Например, при постоянном градиенте межплоскостного расстояния, направленном вдоль оси $Z$, траектории нейтронов в кристалле описываются уравнением [12] (для малых деформаций, когда искажения $\Delta d / d$ много меньше брэгговской ширины $\Delta \lambda / \lambda)$ :

$$
\frac{\partial^{2} z}{\partial y^{2}}= \pm \frac{c^{2}}{2 m_{0}} g \xi
$$

где $c \equiv \tan \theta_{\mathrm{B}}, m_{0} \equiv 2 d F_{g} / V_{c}-$ „масса“ Като $\left(F_{g}-\right.$ структурная амплитуда рассеяния нейтрона, $V_{c}-$ объем элементарной ячейки кристалла, $d-$ межплоскостное расстояние), $g=2 \pi / d-$ вектор обратной решетки, $\xi$ - величина деформации кристалла $\left(d=d_{0}(1+\xi z)\right)$, знак \pm в (1) соответствует двум разным блоховским волнам, возбуждаемым в кристалле. Правая часть уравнения (1) пропорциональна $c^{2}=\tan ^{2} \theta_{\mathrm{B}}$. Величина $c$ может достигать $(10-30)$ при $\theta_{\mathrm{B}} \sim(84-87)^{\circ}$, и влияние малых деформаций на траекторию нейтрона может быть усилено на 2-3 порядка по сравнению с обычными углами дифракции $\left(\theta_{\mathrm{B}} \sim 45^{\circ}\right)$.

Действие внешней силы $F_{e x t}$ на нейтрон в кристалле вдоль вектора обратной решетки $g \| Z$ (рис. 1) эквивалентно линейной деформации кристалла с градиентом $\xi_{f}=\frac{F_{e x t}}{2 E_{n}}$, где $E_{n}-$ энергия нейтрона. Отклонение от брэгговского условия для нейтрона в кристалле во внешнем поле происходит за счет изменения его длины волны.

Уравнение движения нейтрона в кристалле при действии такой силы

$$
\frac{\partial^{2} z}{\partial y^{2}}= \pm \frac{c^{2} g}{2 m_{0}} \frac{F_{e x t}}{2 F_{n}}
$$

Если сравнить его с уравнением движения свободного нейтрона в том же поле

$$
\frac{\partial^{2} z}{\partial y^{2}}=\frac{F_{e x t}}{2 E_{n}}
$$

то можно увидеть, что они отличаются фактором усиления

$$
K_{e}= \pm \frac{c^{2} g}{2 m_{0}}
$$

Письма в ЖТФ, 2017, том 43, вып. 5 


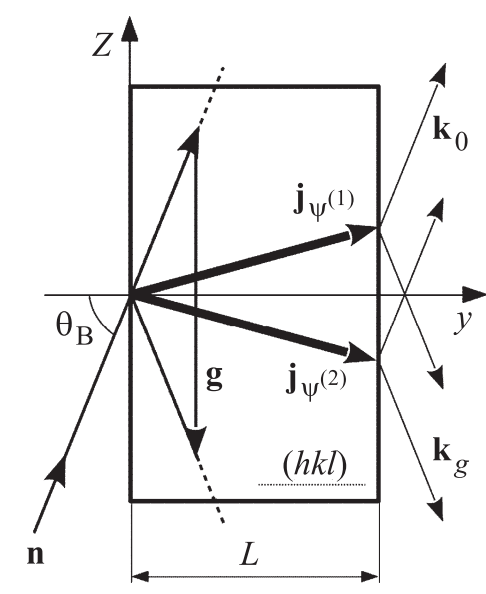

Рис. 1. Симметричный случай дифракции по Лауэ в ограниченном кристалле: $\mathbf{j}_{\psi^{(1)}}$ и $\mathbf{j}_{\psi^{(2)}}-$ вектора плотности потока нейтронов („траектории Като“) для двух блоховских волн, возбуждаемых в кристалле при небольших в пределах брэгговской ширины отклонениях от условия Брэгга; $\mathbf{g}$ - вектор обратной решетки; $\mathbf{n}$ - падающий на кристалл пучок.

который зависит от угла Брэгга как $\tan ^{2} \theta_{\mathrm{B}}$. Численное значение фактора $K_{e}$ для плоскости (220) кристалла кремния равно $K_{e}^{(220)}= \pm 2 \cdot 10^{5} c^{2}$, так что для $\theta_{\mathrm{B}}=86^{\circ}(c=15)$ получается $K_{e}^{(220)}= \pm 4.5 \cdot 10^{7}$.

Эффект дифракционного усиления отклонения направления нейтрона внутри кристалла при малом изменении его угла падения на кристалл хорошо известен [13] и может достигать $\sim 10^{5}-10^{6}$, однако имеется дополнительный фактор, связанный с большими углами дифракции, пропорциональный $\tan ^{2} \theta_{\mathrm{B}}$. Его появление обусловлено тем, что при увеличении угла дифракции время пребывания нейтрона в кристалле растет как $\tan \theta_{\mathrm{B}}$ [14]. Таким образом, появляется новая возможность усиления воздействия внешних сил на дифрагирующий нейтрон при больших углах дифракции. Ее можно использовать, например, для изучения гравитационной силы или силы, обусловленной наличием заряда нейтрона при приложении внешнего электрического поля.

Для обнаружения искривления траектории нейтрона внутри кристалла предлагается использовать двухкристальную геометрию экспе-

Письма в ЖТФ, 2017, том 43, вып. 5 


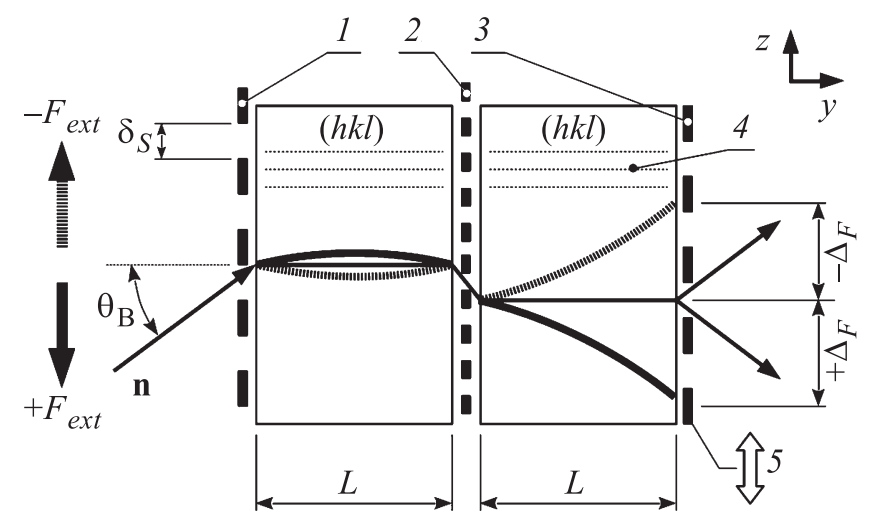

Pис. 2. Схема эксперимента с прямой коллимацией нейтронного пучка: 1,2,3 - входная, промежуточная и выходная (подвижная) щели, 4 - кристаллографические плоскости, 5 - направление движения выходной щели 3.

римента (рис. 2). Идея заключается в прямой коллимации пучка нейтронов, прошедших первый кристалл, двумя щелями и сканированием выходной щелью с целью определения пространственного смещения пучка по выходной грани второго кристалла. Наличие внешней силы приведет к искривлению траекторий нейтрона в кристаллах и, как следствие, к пространственному смещению нейтронного пучка на выходной грани второго кристалла на величину

$$
\Delta_{F}(1,2)= \pm \frac{\pi c^{2} L^{2}}{m_{0} d E_{n}} F_{\text {ext }} \equiv \pm \Delta_{F}
$$

где знак \pm соответствует двум различным блоховским волнам, возбуждаемым в кристалле. Важной характеристикой установки является ее чувствительность к внешней силе, действующей на нейтрон. Определим ее как величину силы $W_{F}$, при которой нейтронный пучок смещается по выходной поверхности кристалла на размер коллимирующей щели. Из уравнения (2) нетрудно получить

$$
W_{F}=\frac{m_{0} d E_{n}}{\pi c^{2} L^{2}} \delta_{s}
$$

Оценки показывают, что, например для плоскости (220) кристалла кремния размерами $L=10 \mathrm{~cm}$, при размере щелей $\delta_{s}=1 \mathrm{~mm}$ и угле

Письма в ЖТФ, 2017, том 43, вып. 5 
дифракции $\theta_{\mathrm{B}}=86^{\circ}$

$$
W_{F}=3 \cdot 10^{-13} \mathrm{eV} / \mathrm{cm}=3 \cdot 10^{-4} m_{n} g,
$$

так что за 100 дней работы реактора для существующих потоков холодных нейтронов можно накопить $10^{9}$ событий и достичь чувствительность к изменению внешней силы

$$
\sigma\left(F_{\text {ext }}\right) \sim 10^{-17} \mathrm{eV} / \mathrm{cm} \cong 10^{8} m_{n} g .
$$

Данная методика описана в работе [1], анализ возможных систематических эффектов проведен в $[15,16]$. Был проведен [17] тест чувствительности установки в однощелевом варианте. К сожалению, увеличение угла Брэгга в этом варианте из-за низкой светосилы установки было невозможно.

В настоящей работе для увеличения счетности была использована многощелевая геометрия (рис. 2). Размеры щелей коллимирующей системы были выбраны аналогично [17]: на входе первого кристалла $16 \mathrm{~mm}$ с периодом $32 \mathrm{~mm}$, между кристаллами $8 \mathrm{~mm}$ с периодом $16 \mathrm{~mm}$, подвижная щель на выходе из второго кристалла $16 \mathrm{~mm}$ с периодом $32 \mathrm{~mm}$. При этом контраст зависимости интенсивностей как отраженной, так и прямой волн от положения выходной щели должен был быть $50 \%$, что и наблюдалось при $\theta_{\mathrm{B}} \sim 76^{\circ}$ (рис. $3, a$ ). На рис. $3, a$ наблюдается достаточно большой фон, часть из которого имеет дифракционную природу (от волн, распространяющихся в кристалле по другим траекториям). Учет его проводился расчетным путем.

На рис. 3, $b$ приведена зависимость величины контраста от угла дифракции. Видно, что увеличение угла Брэгга приводит к уменьшению контраста. Величина пространственного уширения линии $W$, вызвавшего такое падение контраста, приведена на рис. $3, c$. Из (1) следует, что смещение пучка по выходной поверхности за счет деформации кристалла растет как $\operatorname{tg}^{2} \theta_{\mathrm{B}}$. Если предположить, что размытие картины обусловлено деформацией кристалла, то величина градиента деформации кристалла $\xi$, приводящая к такому искажению траектории нейтрона, равна $\xi_{\max }=6 \cdot 10^{-10} \mathrm{~cm}^{-1}$. Это означает, что при размере кристалла в $\sim 20 \mathrm{~cm}$ разброс межплоскостных расстояний находится на уровне $\Delta d / d \sim 10^{-8}$. Сплошная кривая на рис. $3, c-$ результат аппроксимации в таком предположении. Эта оценка является довольно грубой, так как для размытия наблюдаемой экспериментальной картины необходимо,

Письма в ЖТФ, 2017, том 43, вып. 5 

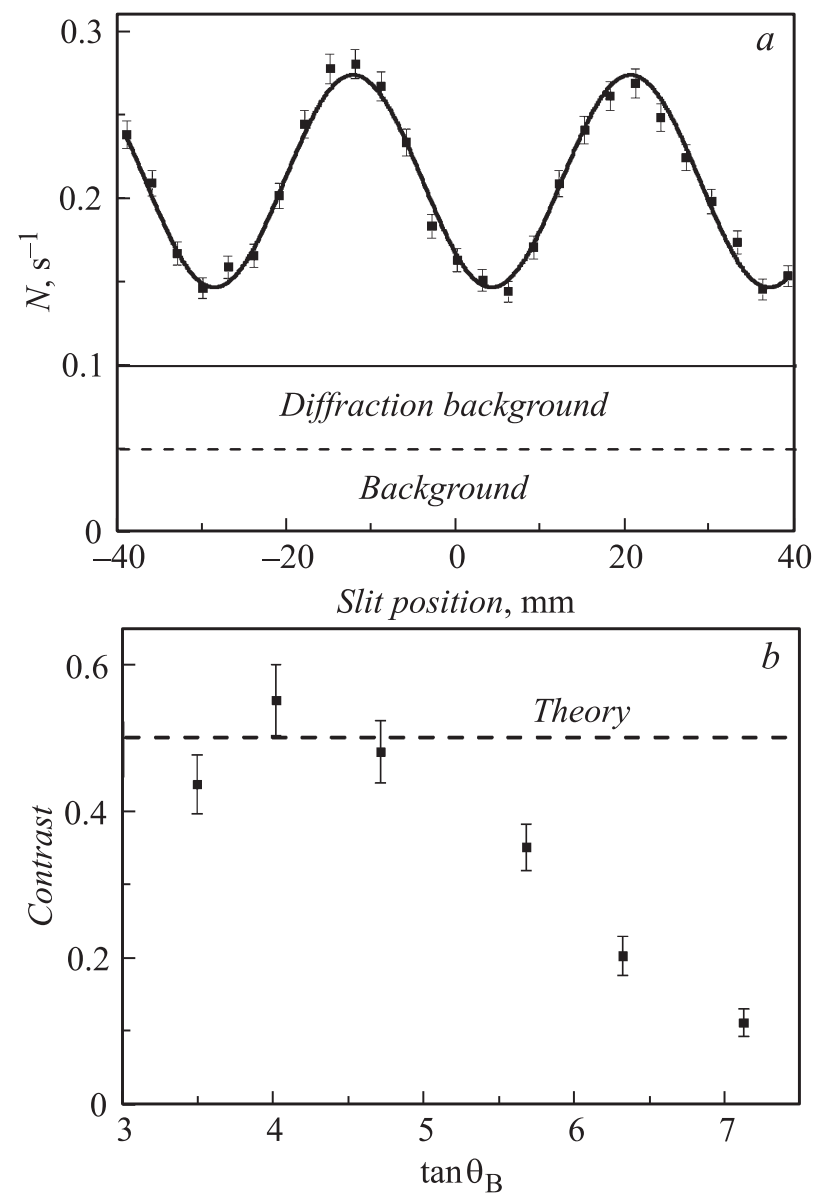

Рис. 3. Экспериментальные данные: $a-$ зависимость интенсивности от положения щели на выходной поверхности кристалла при угле дифракции $76^{\circ}$; $b$ - зависимость контраста (см. $a$ ) от угла дифракции; $c-$ зависимость пространственного разрешения от угла дифракции (сплошная кривая - аппроксимация квадратичной зависимостью); $d-$ зависимость фазы зависимости (a) от угла дифракции.

6 Письма в ЖТФ, 2017, том 43, вып. 5 

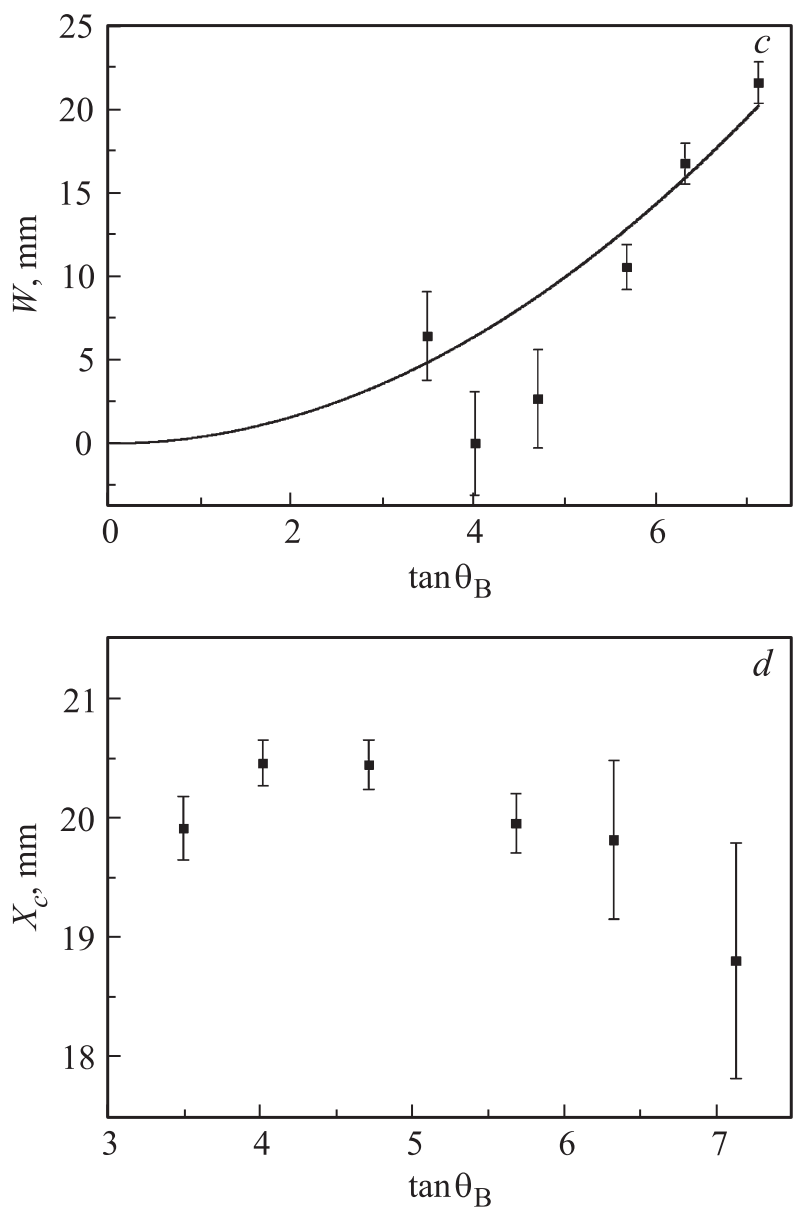

Рис. 3 (продолжение).

чтобы величина $\xi$ менялась для различных траекторий нейтрона в кристалле от 0 до $\xi_{\max }$, а наличие постоянного градиента на уровне даже существенно большем, чем $\xi_{\max }$, приведет не к размытию картины, а лишь к ее пространственному смещению, чего не наблюдалось (рис. 3, d).

Письма в ЖТФ, 2017, том 43, вып. 5 
Таким образом, обнаружен эффект уменьшения пространственного разрешения дифракционной установки для проведения эксперимента по проверке принципа эквивалентности для нейтрона при углах Брэгга $>78^{\circ}$, причина которого до конца не выяснена. Он в несколько раз снижает чувствительность установки, хотя чувствительность тем не менее остается на рекордном уровне. Для устранения эффекта требуются дополнительные исследования на интенсивном пучке холодных нейтронов. В настоящее время такой эксперимент готовится на реакторе ИЛЛ (Гренобль, Франция).

Данная работа поддержана Министерством образования и науки РФ в рамках задания 3.329.2014/К.

\section{Список литературы}

[1] Voronin V.V., Fedorov V.V., Kuznetsov I.A. et al. // Phys. Procedia. 2011. V. 17. P. 232-238.

[2] Adelberger E.G., Gundlach J.H., Heckel B.R. et al. // Prog. Part. Nucl. Phys. 2009. V. 62. P. $102-134$.

[3] Davies P.C.W. // Class. Quantum Grav. 2004. V. 21. N 11. P. 2761-2772.

[4] Accioly A., Paszko R. // Adv. Studies Theor. Phys. 2009. V. 3. N 2. P. 65-78.

[5] Khoury J., Weltman A. // Phys. Rev. Lett. 2004. V. 93. Iss. 17. P. 171104.

[6] Pokotilovskii Yu.N. // Письма в ЖЭТФ. 2012. Т. 96. В. 12. С. 841-843.

[7] Schmiedmayer J. // Nucl. Instr. Meth. A. 1989. V. 284. Iss. 1. P 59-62.

[8] Франк А.И., Гелтенборт П., Жентшель М.В и др. // Письма в ЖЭТФ. 2007. T. 86. B. 4. C. 255-259.

[9] Eotvos R.V., Pekar D., Fekete E. // Ann. Phys. (Leipzig). 1922. V. 68. P. 11-66.

[10] Braginsky V.B., Panov V.I. // Zh. Eksp. Teor. Fiz. 1971. V. 61. N 3. P. 873-879.

[11] Kato N. // J. Phys. Soc. Japan. 1964. P. 971-985.

[12] Alexeev V.L., Lapin E.G., Leushkin E.K. et al. // ЖЭТФ. 1988. T. 94. В. 8. P. 371-383.

[13] Zeilinger A., Shull C.G., Horne M.A. et al. // Phys. Rev. Lett. 1986. V. 57. P. 3089-3092.

[14] Воронин В.В., Лапин Е.Г., Семенихин С.Ю., Федоров В.В. // Письма в ЖЭТФ. 2000. Т. 71. В. 2. С. 110-115.

[15] Воронин В.В., Брагинеи Ю.П., Вежллев Е.О. и др. Препринт ПИЯФ-2849. 2010. $28 \mathrm{c}$.

[16] Воронин В.В., Брагинеи, Ю.П., Кузнецов И.А. Препринт ПИЯФ-2827. 2009. $24 \mathrm{c}$.

[17] Vezhlev E.O., Voronin V.V., Kuznetsov I.A. et al. // Phys. Part. Nucl. Lett. 2013. V. 10. Iss. 4. P. 357-360.

$6^{*}$ Письма в ЖТФ, 2017, том 43, вып. 5 